\title{
The Role of Adjuvant Chemotherapy for Localized Squamous Cell Esophageal Cancer: Current Japanese Standard and the Unending Role of the Drawing Board
}

\author{
Yuko Kitagawa, $\mathrm{MD}^{1}$, Nobutoshi Ando, $\mathrm{MD}^{2}$, Kenichi Nakamura, $\mathrm{MD}^{3}$, Taro Shibata, $\mathrm{MD}^{3}$, and \\ Haruhiko Fukuda, MD $^{3}$ \\ ${ }^{1}$ Department of Surgery, Keio University School of Medicine, Tokyo, Japan; ${ }^{2}$ Department of Surgery, Tokyo Dental \\ College, Ichikawa General Hospital, Ichikawa, Japan; ${ }^{3}$ Japan Clinical Oncology Group Data Center, Tokyo, Japan
}

Esophageal cancer is the sixth most common cause of death for men and, among the various gastrointestinal malignancies, it is considered to possess a relatively high malignant potential. Major causes of the difficulties in the management of this clinical entity are largely related to its clinicopathological characteristics, particularly the high frequency of complex patterns of lymph node metastasis.

As a result of intense discussion beginning approximately 30 years ago, radical esophagectomy with threefield lymphadenectomy has become established in leading institutes in Asia since the mid 1980 s. Although relatively acceptable long-term outcomes have been reported, one can claim by no means that substantial evidence of survival benefit has been proved by large-scale, randomized, controlled trials (RCT). Even in the high-volume centers in Asia, further improvement of 5-year survival rates by surgery alone appears to be extremely unlikely.

Therefore, optimization of multimodal treatments for localized and resectable clinical stage II/III esophageal cancer is one of the most important topics in this field. Several clinical trials in the west have demonstrated the superiority of preoperative chemoradiation therapy compared with surgery alone. ${ }^{1}$ However, it must be admitted that one of the most conspicuous features, and one that in the minds of many constitutes a critical limitation of the above-mentioned western studies, has been the extremely poor outcome of the surgery-alone groups. On the other hand, Japanese surgeons believe that the relatively

(C) Society of Surgical Oncology 2012

Published Online: 7 March 2012

Y. Kitagawa, MD

e-mail: kitagawa@a3.keio.jp acceptable local tumor control by transthoracic radical esophagectomy obviates the need for preoperative radiation therapy, especially because the inevitable fibrotic changes induced by radiation would negatively affect the quality of the surgical approach. Therefore, many Asian physicians treating patients with esophageal squamous cell carcinoma hesitate to apply directly the presently available results of "Western evidence" to "Eastern" esophageal cancer, in which the environment of latter is different from the former.

The Japan Clinical Oncology Group (JCOG) has conducted multicenter, multimodality, prospective, clinical trials for the treatment of esophageal cancer for more than 30 years, giving full regard to these considerations. Recently, a JCOG study (JCOG9907) demonstrated significantly better overall survival after preoperative chemotherapy with two courses of cisplatin plus 5-fluorouracil followed by surgery compared with postoperative chemotherapy for resectable cStageII/III thoracic squamous cell esophageal cancer. ${ }^{2}$ Since publication of the results of the JCOG9907 study, preoperative chemotherapy followed by radical esophagectomy has been accepted as the standard therapeutic approach to resectable cStage II/III esophageal cancer in Japan.

On the other hand, it cannot be denied that the JCOG9907 study has aroused several controversies among some extremely knowledgeable experts who are seeking to examine and interpret the present study as much scientific rigor as possible. In a recent editorial article, Ajani et al. mentioned problems that they noted in the study design of JCOG9907. ${ }^{3}$ As representatives of the JCOG Esophageal Cancer Study Group, we would like to respond to their specific criticisms to encourage further understanding 
regarding the current Japanese standard, among both western and non-western investigators. One of their major points of criticism is that in the postoperative treatment arm, pathologically node-negative patients did not receive chemotherapy because a previous RCT comparing postoperative chemotherapy vs. surgery alone (JCOG9204) did not show any benefit for adjuvant chemotherapy in pathologically node-negative patients. ${ }^{4}$ Even though this finding came from a subset analysis, we considered that there was no sufficient reason to add toxic postoperative chemotherapy to $\mathrm{pN} 0$ patients, because the RCT results had already demonstrated that more than $70 \%$ of this particular group would achieve 5-year, disease-free survival. Consequently postoperative chemotherapy with cisplatin plus 5-fluorouracil except for $\mathrm{pN} 0$ patients became the standard treatment for Japanese patients. We must most emphatically stress that this study was by no means a mere comparison between preoperative and postoperative chemotherapy but a comparison among multimodality treatments with curative esophagectomy, including chemotherapy, as only one component of the whole.

In the editorial, reference was made to comparison of apples and oranges. Although we agree that that kind of a comparison can be made at the most elemental level, we must reiterate our statements in the previous paragraph: 1) results of an RCT showed lack of justification for giving postoperative chemotherapy to patients demonstrated pathologically to have no recognizable lymph node metastasis, and 2) it was not a simplistic comparison between preoperative and postoperative chemotherapy. Rather, it represents a more complexly designed plan, deliberately worked out at the drawing board, as part of the process toward designing the best possible therapeutic approach to this entity. This was a more holistic multimodality treatment development study with the option of inclusion of scheduled chemotherapy and the possibility of referring to pathologic findings of resected specimens to try to determine the optimal treatment for these patients. In actual fact, the 5-year overall survival of $\mathrm{pN0}$ patients who underwent no postoperative chemotherapy in the postoperative chemotherapy group was not excessively poor (64\%), whereas that of $\mathrm{pN} 1$ patients who were unable to undergo postoperative chemotherapy was dismal $(0 \%)$ as described in the discussion session. Survival data for $\mathrm{pN} 0$ patients with no postoperative chemotherapy was not the main factor for the poor outcome in the postoperative chemotherapy group; rather $\mathrm{pN} 1$ status with no postoperative chemotherapy for various reasons was the major factor reducing overall survival in the postoperative chemotherapy group. The completion rate of two courses of chemotherapy was $75 \%$ (81/108 patients) in the postoperative chemotherapy group, and it was $88 \%$ (140/ 159 patients) in the preoperative chemotherapy group as described in the figure in the original paper. Therefore, the study design with no postoperative chemotherapy for $\mathrm{pN} 0$ patients did not confuse the interpretation or conclusions of the present study.

Although there was a discrepancy between progressionfree survival and overall survival, with overall survival showing greater preponderance than progression-free survival, the Data and Safety Monitoring Committee recommended early publication. Even though the preoperative chemotherapy group showed better progression-free survival (but not with statistical significance), the primary endpoint progression-free survival did not meet the prespecified stopping criterion. However, be that as it may, a large difference between the two groups was observed in overall survival $(P=0.01$, unstratified logrank test; hazard ratio, 0.64; 95\% CI, 0.45-0.91).

Ajani et al. also were concerned whether this unstratified analysis included those patients who were not treated in the postoperative arm. We thank them for making this point. Our text should have made this clearer: of course, those who were not treated in the postoperative arm were included in the unstratified analyses. In stratified analysis, an overall test statistic is calculated by summing the log-rank statistics and corresponding variances obtained within each of the independent strata. ${ }^{5}$ On the other hand, a crude test statistics is calculated in unstratified analysis. Therefore, unstratified analyses are not meant to exclude some patients from analyses. Those analysis methods were prespecified in the Statistical Analysis Plan. Ajani et al. pointed out the importance of cancer-specific survival to interpret the data of the present study. Needless to say, to censor the other types of death other than cancer death and calculate the Kaplan-Meier estimates would involve a major statistical problem. ${ }^{6,7}$ Generally, it is difficult to interpret a cancer-specific survival because of "competing risks" even with a specific analysis method, so cancer-specific survivals are generally not calculated in JCOG studies.

Although the current Japanese standard for resectable cStageII/III esophageal squamous cell carcinoma is preoperative chemotherapy with cisplatin plus 5-fluorouracil, subgroup analysis has shown survival benefit in cStage III to be insufficient. Therefore, development of more effective preoperative treatment is required. Now, JCOG is preparing to conduct a three-arm randomized, controlled trial comparing preoperative chemoradiation therapy with cisplatin plus 5-fluorouracil and preoperative chemotherapy with docetaxel in addition to cisplatin and 5-fluorouracil (DCF) to standard preoperative treatment with cisplatin plus 5-fluorouracil. This study should be a significant milestone for surgical oncology in examining the possible additive efficacy and safety of preoperative chemoradiation, which is the current standard in the west.

Individualized therapy based on biomarkers will be a key factor in all aspects of clinical oncology. In the field of treatment of resectable cStage II/III esophageal squamous 
cell cancer, identification of reliable biomarkers to predict response for neoadjuvant treatment is absolutely critical and is the next target of basic and clinical investigations.

In their important editorial, Ajani and Swisher indicate in their title the importance of "We should go back to the drawing board!" We could not agree more, although not in the sense of having to start from scratch, but in the sense that even though we have come a long way in the treatment of this disease, just as a remarkable edifice requires blueprint after blueprint, we will need to continue defining and refining our strategy.

ACKNOWLEDGMENT The authors are indebted to Prof. J. Patric Barron of the International Medical Communications Center of Tokyo Medical University for his review of this manuscript.

\section{REFERENCES}

1. Gebski V, Burmeister B, Smithers BM, et al. Survival benefits from neoadjuvant chemoradiotherapy or chemotherapy in oesophageal carcinoma: a meta-analysis. Lancet Oncol. 2007;8:226-34.
2. Ando N, Kato H, Igaki H, Shinoda M, et al. A randomized trial comparing postoperative adjuvant chemotherapy with cisplatin and 5-fluorouracil versus preoperative chemotherapy for localized advanced squamous cell carcinoma of the thoracic esophagus (JCOG9907). Ann Surg Oncol. doi:10.1245/s10434-011-2049-9

3. Ajani JA, Stephen, Swisher SG. Preoperative chemotherapy for localized squamous cell carcinoma of the esophagus? We should go back to the drawing board! Ann Surg Oncol. doi:10.1245/s10434011-2101-9

4. Ando N, Iizuka T, Ide H, et al. Surgery plus chemotherapy compared with surgery alone for localized squamous cell carcinoma of the thoracic esophagus: a Japan Clinical Oncology Group Study JCOG9204. J Clin Oncol. 2003;21:4592-6.

5. Kalbfleisch JD, Prentice RL. The statistical analysis of failure time data, 2nd edn. Hoboken: Wiley, 2002:22-3.

6. Green S, Benedetti J, Crowley J. Competing risks. In: Clinical trials in oncology, 2nd edn. Boca Raton: Chapman and Hall/CRC, 2003:188-94.

7. Tsiatis AA. Competing risks. In: Armitage P, Colton T, eds. Encyclopedia of biostatistics, $2^{\text {nd }}$ edn. Chichester: Wiley, 2005:1025. 